\title{
MULTIVARIABLE STABILITY MARGIN CALCULATION WITH UNCERTAIN CORRELATED PARAMETERS
}

\author{
Athanasios Sideris \\ California Institute of Technology \\ Dept. of Electrical Engineering \\ Pasadena, CA 91125
}

\author{
Raymond R. E. de Gaston \\ University of Southern California \\ Dept. of Electrical Engineering-Systems \\ Los Angeles, Ca 90089-0781
}

\begin{abstract}
An algorithm is developed for the computation of the multivariable stability margin in the case of real uncertain system parameters which can be related to each other.
\end{abstract}

\section{Introduction}

In the design of feedback control systems it is important that certain properties established for the nominal model employed to describe the physical process to be controlled are maintained in the face of model uncertainty. The feedback system of Fig. 1, where $P(s)$ represents the plant and $C(s)$ the controller, is called robustly stable when it is closed-loop stable for the nominal plant model and also remains stable for a given class of model perturbations. Therefore it is very important to have tools that determine the robustness properties of a design with respect to the class of model perturbations considered.

There are well-khown conditions for the so-called case of unstructured model uncertainty, that is, when only a frequency dependent norm bound on the plant perturbation is assumed $[1,2]$.

However, it is usually the case that plant uncertainty is structured, i. e., there is more information available about it than just a magnitude bound. Safonov [3] considered a canonical block diagonal perturbation system obtained by rearranging uncertainty blocks from various plant locations into a block diagonal form (see Fig. 2). In this setting the condition for stability is given in terms of the multiloop stability margin $k_{m}$, which is defined as

$$
k_{m} \stackrel{\text { def }}{=} \min _{\Delta}\{k \in[0, \infty) \mid \operatorname{det}(I-k \Delta H)=0\}
$$

where

$$
\Delta \stackrel{\text { def }}{=} \operatorname{diag}\left[\Delta_{1}, \Delta_{2}, \ldots, \Delta_{n}\right]
$$

and the $\Delta_{i}$ 's are norm bounded. By introducing stable and minimum phase weighting factors, which can be incorporated in the nominal block $H(s)$, we can assume with no loss of generality that $\left\|\Delta_{i}\right\|<1$ for all $\omega$. Then robust stability is assured if and only if $k_{m}>1$. Clearly $k_{m}$ is a function of frequency and the nominal plant and also depends on the block uncertainty structure. Doyle [4] considers $\mu=1 / k_{m}(H)$ and computes it exactly for three or less blocks in $\Delta$ and gets bounds on it in more general cases. Now closed-loop stability is assured if and only if $\mu(H)<1$ The previous results prove to be conservative if one or more of the $\Delta_{i}$ 's are real uncertain parameters. de Gaston [5] and de Gaston and Safonov [6] derive an algorithm to compute $k_{m}$ to any desired degree of accuracy in the case that the $\Delta_{i}$ 's in Fig. 2 are real bounded scalars. This technique can be modified to handle complex $\Delta_{i}$ 's as well, which provides a very general result. However in the approach of $[5,6]$ the $\Delta_{i}$ 's are restricted to be independent of each other and this is often not the case even for simple examples (see section 3 ).

In this paper it is shown how the multiloop stability margin can be computed in the most general case of correlated real uncertain plant parameters. Our results can be also used to analyze the integrity of the design with respect to sensors and/or actuators failures. We can simply consider the relevant gains as uncertain parameters that can assume the zero value.

An outline of the remainder of the paper follows. In Section 2 the computation of $k_{m}$ in the case of uncorrelated $\Delta_{i}$ 's is treated in similar spirit with $[5,6]$. A new simpler proof of the convergence of upper and lower bounds on $k_{m}$ derived in $[5,6]$ is given here. In Section 3 the main results of the paper are presented. The problem with uncertain corellated parameters is transformed to one with uncorrelated parameters with some additional canonical constraints among them. Modifications to the previous algorithm are introduced to accomodate these constraints and indeed to take advantage of them to reduce the computations. In Section 4 we discuss an example. Finally in Section 5 the main points of the paper are summarized.

2. Computation of $k_{m}$ for Uncorrelated Uncertain Parameters

In the following we give a concise treatement of the algorithm for the computation of the multiloop stability margin $k_{m}$, defined by (1.1), in the case of uncorrelated uncertain parameters $\{5,6]$. In this algorithm we can identify two mechanisms. One is used to produce upper and lower bounds on $k_{m}$. The other is used to refine these bounds so that an increasing sequence $l_{r}$ of lower bounds and a decreasing sequence $u_{r}$ of upper bounds that converge to $k_{m}$ are produced. We discuss these two mechanisms in turn.

The derivation of upper and lower bounds on $k_{m}$ is based on the following theorem in Zadeh and Desoer [7] regarding multilinear functions on $R^{n}$. A function $f\left(\delta_{1}, \ldots, \delta_{n}\right): D \subset$ $R^{n} \longrightarrow \mathcal{C}$ is called multilinear if and only if it is affine in each of the $\delta_{i}$ 's.

Propasition 1 [7]

Let $f(\delta)$ be a multilinear function of $\delta=\left(\delta_{1}, \ldots, \delta_{n}\right)^{T} \in D \subset$ $R^{n}$. Then every line segment of $D$ parallel to a coordinate axis is mapped to a line segment on the complex plane.

As an immediate consequence of this proposition and by the definition of the convex hull of a region $X \in \mathcal{C}$ as the smallest convex set $c o(X) \in \mathscr{C}$ which contains $\chi$, we have the following:

Corollary 1

Let $f(\delta)$ be a multilinear function on $D \in R^{n}$ where $D$ is convex and compact. Consider the plane sets

$$
f(D) \stackrel{\text { def }}{=}\{z \in \mathcal{C} \mid z=f(\delta) \text { for some } \delta \in D\}
$$




$$
f(\vartheta D) \stackrel{\text { def }}{=}\{z \in \mathscr{C} \mid z=f(\delta) \text { for some } \delta \in \vartheta D\}
$$

where $\vartheta D$ is the boundary of $D$. Then $f(D)$ is contained in $\operatorname{co}(f(\vartheta D))$.

Proof: Every point of $D$ lies on a line segment parallel to one of the coordinate axes with ends on $\vartheta D$ by convexity. Since the image of this line segment is again a line segment joining two points on $f(\vartheta D)$ by Proposition 1, the assertion follows.

By taking $D$ to be a hypercube in $R^{n}$, we can establish the following lemma. We introduce first some notation.

Notation

$D^{\text {def }}=\left\{\delta=\left\{\delta_{3}, \ldots, \delta_{n}\right]^{T} \in R^{n} \mid-1 \leq \delta_{i} \leq 1, i=1, \ldots, n\right\}$

$\Delta \stackrel{\text { def }}{=} \operatorname{diag}\left\{\delta_{1}, \delta_{2}, \ldots, \delta_{n}\right\}$ with $-1 \leq \delta_{i} \leq 1, i=1, \ldots, n$

$V \stackrel{\text { def }}{=}\left\{V_{i}=\left|v_{i 1}, \ldots, v_{i n}\right|^{r} \in R^{n} \mid v_{i j}=1\right.$ or $\left.-1, j=1, \ldots, n, i=1, \ldots, 2^{n}\right\}$

$\mathrm{H}(\mathrm{s})$ : stable transfer function matrix

$\operatorname{det}[I-k D h(j \omega)] \stackrel{\operatorname{def}}{=}\{z \in \mathcal{K} \mid z=\operatorname{det}[(I-k \Delta H(j \omega)]$ for $\delta \in D\}$

$\operatorname{det}[I-k V h(j \omega)] \stackrel{\text { det }}{=}\{z \in f \mid z=\operatorname{det}[(I-k \Delta H(j \omega)]$ for $\delta \in V\}$

Lemma 1

Consider for any fixed $\omega$ the mapping $\operatorname{det}(I-k \Delta H(j \omega))$, with $D, \Delta$, and $H(s)$ as defined above. Then(see Fig. 3a):

$$
\operatorname{det}[I-k D H(j \omega)] \in \operatorname{co}\{\operatorname{det}[I-k V H(j \omega)]\}
$$

Proof: The result follows immediately from Corollary 1 , since $\operatorname{det}[I-k \Delta H(j \omega)]$ is multilinear with respect to the $\delta_{i}$ 's and since $c o\{\operatorname{det}[I-k \vartheta D H(j \omega)]\} \in \operatorname{co}\{\operatorname{det}[I-k V H(j \omega)]\}$.

The procedure for obtaining upper and lower bounds on $k_{m}$ is next given by way of the following theorem.

\section{Theorem 1}

We consider the mapping $\operatorname{det}[I-k \Delta H(j \omega)]$ and the complex region $\operatorname{co}\{\operatorname{det}[I-k V H(j \omega)]\}$ defined above. We increase $k$ from $k=0$ to $k=l$ so that this region intercepts the origin for first time. If this does not occur for $k<\infty$ then it holds $k_{m}(j \omega)=\infty$. Otherwise for $k=l$ we can either identify one vertex of $D$ which is mapped onto the origin, or two vertices $V_{i}$ and $V_{j}$ so that the origin lies on the line segment joining their images. In the former case $k_{m}(j \omega)=l$. In the latter case $V_{i}$ and $V_{j}$ are called critical vertices* and $l$ is a lower bound on $k_{m}(j \omega)$ (see Fig $3 a$ ). When critical vertices are defined, we consider all paths from $V_{i}$ to $V_{j}$ formed from edges of the hypercube $D$ (defined as vertex paths ${ }^{*}$ ) and we increase $k$ to $k=u$ so that the image of one such path first intercepts the origin(see Fig. 3b). If this does not occur for $k<\infty$ we set $u=\infty$. Then $u$ is an upper bound on $k_{m}(j \omega)$.

Proof: It follows from Lemma 1 that $l \leq k_{m}$. Thus if $l=\infty$ then $k_{m}(j \omega)=\infty$. In the case that some vertex of $D$ is mapped on the origin, then definition (1.1) yields $k_{m}(j \omega)=$ l. If there exists $u<\infty$ as in the theorem, then it holds $k_{m}(j \omega) \leq u$, since all points of a vertex path belong to $D$. Otherwise $u=\infty$, and $k_{m}(j \omega) \leq u$ is trivially true.

The refining of the bounds of Theorem 1 is accomplished in the following theorem. An algorithmic procedure is given in which $D$ is successively subdivided, so that if $\delta^{*}$ is a point of $D$ which satisfies det $\left[I-k_{m} \Delta H(j \omega)\right]=0$, a sequence of subdomains is produced which converges to $\delta^{*}$. At each step of the algorithm lower and upper bounds $l_{r}$ and $u_{r}$ are produced which converge to $k_{m}(j \omega)$. In case that $k_{m}(j \omega)=\infty$,

* Note that in $[5,6]$ critical vertices and vertex paths were defined as the images of what we defined them here. it can be shown that the sequence of lower bounds grows unbounded. More details and examples can be found in $[5,6]$, but the proof given here is original.

Theorem 2

Let $D, H(s), \operatorname{det}[I-k \Delta H(j \omega)]$, and $\operatorname{det}[I-k V H(j \omega)]$ be as defined above. Then there exists a decomposition

$$
D=D_{1} \cup D_{2}
$$

$D_{1} \stackrel{\text { def }}{=}\left\{\delta \in R^{n} \mid 0 \leq \delta_{i} \leq 1, i=1, p-1, p+1, \ldots, n\right.$ and $\left.-1 \leq \delta_{p} \leq 0\right\}$

$D_{2} \stackrel{\text { def }}{=}\left\{\delta \in R^{n} \mid 0 \leq \delta_{i} \leq 1, i=1, p-1, p+1, \ldots, n\right.$ and $\left.0 \leq \delta_{p} \leq 1\right\}$

so that if we obtain bounds $l$ and $u$ for $D, l_{11}$ and $u_{11}$ for $D_{1}$, and $l_{12}$ and $u_{12}$ for $D_{2}$ as in Theorem 1 and we set $u_{1}=$ $\min \left\{u_{1 i}, u\right\}$, it holds

$$
l<l_{1 i} \leq k_{m} \leq u_{1} \leq u, i=1 \text { or } 2
$$

This decomposition of $D$ can be continued so that at the $r^{\text {th }}$ step we obtain $s(r)$ subdomains of $D$ with $s(r) \leq 2^{r}$ and corresponding bounds $l_{r i}, u_{r i}, i=1, . ., s(r)$, such that if we set $u_{r} \stackrel{\text { def }}{=} \min \left\{u_{r i}, u_{r-1}\right\}$, it holds

$$
l_{r i} \leq k_{m} \leq u_{r} \text {, for some } i=i^{*}, 1 \leq i \leq s(r)
$$

In this manner we obtain sequences $l_{r} \stackrel{\text { def }}{=} l_{r i^{*}}$, and $u_{r}, r=$ $1,2, \ldots$, such that

$$
\begin{gathered}
l_{r} \leq k_{m} \leq u_{r}, r=1,2, \ldots \\
l_{r}<l_{r+1}, \quad r=1,2, \ldots \\
l_{r} \longrightarrow k_{m}, \quad r \longrightarrow \infty \\
u_{r+1} \leq u_{r}, \quad r=1,2, \ldots
\end{gathered}
$$

and if $n \leq 2$ or $\left(k_{m}, \delta^{*}\right)$ is not an isolated real solution of (1.1)

$$
u_{r} \longrightarrow k_{m}, r \longrightarrow \infty
$$

Proof: Let $k$ increase to $k=l$ so that $\operatorname{co}\{\operatorname{det}[I-k V H(j \omega)]\}$ intercepts the origin and that critical vertices $V_{i}, V_{j}$ are defined and $V_{i} \neq V_{j}$ (see Theorem 1). Then there exists at least one decomposition of $D$ as in $(2.8 \mathrm{abc})$ so that $V_{i}$ and $V_{j}$ belong to different halves of $D$. Then by a geometric argument [5], it holds $l_{1 i}>l, i=1,2$ (see Fig. 4). Consider $D_{i^{*}}, i^{*}=1$ or 2 , that contains $\delta^{*}$. Then $l_{1 i *} \leq k_{m}$ and (2.9) follows. Define $l_{1} \stackrel{\text { def }}{=} l_{1 i^{*}}$. We continue this procedure by dividing at the $r^{\text {th }}$ step the subdomains defined at the $(r-1)^{\text {th }}$ step. Note that at each step $\delta^{*}$ is contained in some subdomain $D_{r i *}$ and the corresponding bound satisfies $l_{r i^{*}} \leq k_{m}$. We then define $l_{r} \stackrel{\text { def }}{=} l_{r i *}$ and $u_{r} \stackrel{\text { def }}{=} \min \left\{u_{r i}, u_{r-1}\right\}$. Then (2.10ab) follow.

At the $r^{\text {th }}$ step we need not consider subdomains $D_{r i}$ for which $l_{r i}>u_{r-1}$. The subdomain count obviously satisfies $s(r) \leq 2^{r}$. Property (2.11) follows if we divide subdomains across any one of the edges for which separation of the critical vertices is achieved (see Fig 4). To prove (2.12) consider $\epsilon>0$ and $l$ such that $k_{m}-\epsilon \leq l \leq k_{m}$. Let $l \cdot D \stackrel{\text { def }}{=}\left\{\delta \in R^{n} \mid \delta=\right.$ $\left.l \cdot \delta_{1}, \delta_{1} \in D\right\}$. By the continuity of the mapping function and the compactness of $l \cdot D$, we can assume that by subdividing subdomains fine enough, their images are contained in disks that do not include the origin. This implies that the convex hulls of these images do not contain the origin and thus $l_{r}>l$. 
Since $\epsilon$ is arbitrary, we obtain $l_{r} \rightarrow k_{m}$, as $r \rightarrow \infty$.

Next (2.13) is obvious by construction, and (2.14) can be shown as follows. We can assume that the sequence of subdomains that contain $\delta^{*}$ actually converges to $\delta^{*}$, by inserting if necessary subdivision steps for edges that do not separate critical vertices and are not subdivided otherwise. Let $D_{r}$ be this sequence of subdomains. If $r \leq 2$ or $\left(k_{m}, \delta^{*}\right)$ is not an isolated real solution of (1.1), some vertex path will intercept the origin at a point $\delta_{r}$ in $D_{r}$, as $r \rightarrow \infty$ and for $k=u_{r}$. Obviously $u_{r}$ is an upper bound on $k_{m}$ and satisfies $\operatorname{det}\left[I-u_{r} \Delta_{r} H(j \omega)\right]=0$. Now since $\delta_{r} \rightarrow \delta^{*}$,we obtain $u_{r} \rightarrow k_{m}(j \omega)$ as $r \rightarrow \infty$, by the continuity of the roots of a polynomial equation with respect to its coefficients. Note that $u_{r}$ and $k_{m}$ are the smallest positive real roots of $\operatorname{det}[I-k \delta H(j \omega)]$ for $\delta$ equal to $\delta_{r}$ and $\delta^{*}$ respectively. The proof of Theorem 2 is now complete.

\section{Remarks}

1. It has been observed in computer simulations that only a small number of subdomains need to be further subdivided at every step, because the rest have lower bounds greater than the upper bound of the previous step. This and the fact that computations involve only polynomial evaluations at a small number of points make the algorithm very efficient.

2. If the assumption associated with (2.14) is not satisfied, we cannot generically obtain a finite upper bound. Nevertheless since $\delta^{*}$ is isolated, all subdomains but the ones converging to the ray through $\delta^{*}$ will have an infinite lower bound and can be dropped.

3. The previous procedure produces the stability margin for real parameter variations at any desired frequency. However, due to the real nature of the parameters, $k_{m}(j \omega)$ can be a discontinuous function of $\omega$. Methods on how to determine the discontinuites of $k_{m}(j \omega)$ are currently being examined.

\section{Main Results-Computation of $k_{m}$ in the General Case}

A crusial assumption in the previous section was that the uncertain system parameters were uncorrelated,i. e., there were no functional relationships among the $\delta_{i}$ 's. However, this is usually not the case in practice. For example suppose that the plant is modelled by

$$
P(s)=\frac{1}{s^{2}+2 \zeta \omega_{n} s+\omega_{n}^{2}}
$$

where $\zeta$ and $\omega_{n}$ are uncertain. To bring the closed loop system of Fig. 1 to the canonical diagonal form $\Delta$ of Fig. 2, we have to select as our uncertain parameters $\delta_{1}=\zeta \omega_{n}, \delta_{2}=\omega_{n}^{2}$ or $\delta_{1}=\zeta, \delta_{2}=\omega_{n}, \delta_{3}=\omega_{n}^{2}$, and in both cases the $\delta_{i}$ 's are obviously correlated.

In the case of correlated system parameters, the algorithm of the previous section fails because $\operatorname{det}[I-k \Delta H(j \omega)]$ is not defined on all of the hypercube $D$. Thus this algorithm will produce in general only a lower bound on $k_{m}$. In this section we show how to compute the multiloop stability margin in the most general case. This is accomplished by redefining $k_{m}$ so that the condition $k_{m}>1$ still reflects robust stability, but its computation is now possible by using the algorithm of the previous section properly modified. These modifications are described in the following steps.
Step 1 (Redefinition of $k_{m}$ )

We consider the feedback system of Fig. 1, and we assume that the plant $P(s)$ and the controller $C(s)$ depend on a set of real parameters $\delta_{1}, \delta_{2}, . ., \delta_{n}$. By introducing weigthing factors we can assume that $\left|\delta_{i}\right| \leq 1, \mathrm{i}=1, \ldots, \mathrm{n}$, so that $\delta \stackrel{\text { def }}{=}$ $\left(\delta_{1}, \ldots, \delta_{n}\right)^{T} \in D$. ity.

The following theorem gives conditions for robust stabil-

Theorem 3 (see for example [8])

In the system of Fig. 1, let the closed-loop system charasteric polynomial $p(s ; \delta)$ depend on the parameter vector $\delta \in D$. Then the feedback system is robustly stable (i.e. for all values of the parameters) if and only if

$$
\begin{aligned}
& \text { 1. } p\left(s ; \delta_{0}\right) \text { is stable for some } \delta_{0} \in D \\
& \text { 2. } p(j \omega ; \delta) \neq 0 \text { for all } \delta \in D \text {, and for all } \omega
\end{aligned}
$$

The multivariable stability margin is defined in the context of the previous theorem as follows.

Definition 1

Let $p(s ; \delta)$ be the closed-loop characteristic polynomial and suppose that the nominal closed-loop system is stable, i.e. $p\left(s ; \delta_{0}\right)$ has all its roots in $R e[s]<0$. Consider the function $p(j \omega ; k \delta)$ for each $\omega$, where $k \in[0, \infty)$. Then

$$
k_{m}(j \omega) \stackrel{\text { def }}{=} \text { in }\{\{k \mid p(j \omega ; k \delta)=0 \text { for } \delta \in D\}
$$

We remark that robust stability is again achieved if and only if $k_{m}>1$ for all $\omega$. In this formulation the mapping function $p(j \omega ; k \delta)$ is defined on a hypercube as in the case of uncorrelated parameters. Moreover no system rearrangement is necessary to derive $p(j \omega ; \delta)$. However, the mapping $p(j \omega ; k \delta)$ is not multilinear and Proposition 1 and Lemma 1 supporting the algorithm of the previous section do not hold. This is attended to next.

\section{Step 2 (Modification of the mapping function)}

\section{Lemma 2}

We assume that $p(j \omega ; \delta)$ is a polynomial in each of the $\delta_{i}$ 's. Let $m_{i}$ be the highest degree of $\delta_{i}$ in $p(j \omega ; \delta)$. Consider ficticious variables $\delta_{i 1}, \ldots, \delta_{i m(i)}, i=1, \ldots, n$ and replace in $p(j \omega ; \delta)$ each $\delta_{i}^{p}$ with $\delta_{i 1} \delta_{i 2} \ldots \delta_{i p}$. Let

$$
\begin{gathered}
\bar{D} \stackrel{\text { def }}{=}\left\{\bar{\delta} \stackrel{\text { def }}{=}\left[\delta_{i 1}, . ., \delta_{i m(i)}, . ., \delta_{n 1}, . ., \delta_{n m(n)}\right]^{T} \mid-1 \leq \delta_{i j} \leq 1,\right. \\
i=1, . ., n, j=1, \ldots, m(i)\}
\end{gathered}
$$

Also let

$$
\begin{gathered}
\bar{p}(j \omega ; \bar{\delta}) \stackrel{\text { def }}{=} p(j \omega ; \delta) \\
\bar{p}(j \omega ; k \in \bar{D}) \stackrel{\text { def }}{=}\{\boldsymbol{z} \mid z=\bar{p}(j \omega ; k \bar{\delta}) \text { for } \bar{\delta} \in \bar{D}\}
\end{gathered}
$$

Then $\bar{p}(j \omega ; k \bar{\delta})$ is a multilinear function of $\bar{\delta}$ and $k_{m}$ as defined in (3.2) equals

$$
k_{m} \stackrel{\text { def }}{=} i n f\left\{k \mid \bar{p}(j \omega ; k \bar{\delta})=0 \text { for } \bar{\delta} \in \bar{D} \text { and } \delta_{i,}=\delta_{i t}, i=1, \ldots, n\right\}
$$

Proof: Obvious by the construction of $\bar{p}(j \omega ; \bar{\delta})$. 
We remark that the assumption that $p(j \omega ; \delta)$ is a polynomial in the $\delta_{i}$ 's is flexible enough to cover most practical situations, but also smooth nonlinear functions of the $\delta_{i}$ 's can be approximated on a compact set of $R^{n}$ as closely as desired by a multivariate polynomial [9].

At this point our intention is to exploit the properties of the multilinear function $\bar{p}(j \omega ; \bar{\delta})$ (Proposition 1 ,Lemma 1 ) to compute $k_{m}$. However, Theorems 1 and 2 have to be modified to accomodate for the additional equality canonical constraints:

$$
\delta_{i s}=\delta_{i t}, i=1, \ldots, n
$$

\section{Step 3 (Modification of Theorem 1)}

The part of Theorem 1 regarding the derivation of lower bounds on $k_{m}$ remains essentially the same. However, we have to work harder to obtain an upper bound on $k_{m}$, since in general critical vertices or vertex paths do not satisfy (3.6). To this end we introduce the following definitions.

\section{Definition 2}

A constrained edge (ce) of $\bar{D}$ is a line segment joining two vertices of $\bar{D}$ that satisfy constraints (3.6) and such that if coordinates corresponding to equal variables are identified, these vertices do not differ in more than one coordinates.

\section{Definition 3}

A generalized constrained edge (gce) of $\bar{D}$ is the smallest face of $\bar{D}$ (i.e a part of $\bar{D}$ defined by fixing some of the coordinates at extreme values), that contains a constrained edge.

\section{Definition 4}

We say that the image of a gce of $\bar{D}$ mapped by $\bar{p}(j \omega ; k \bar{\delta})$ is crossed by the origin, if as we increase $k$, both boundary arcs of it joining the images of the two vertices in the gce that satisfy (3.6) are crossed by the origin.

The above definitions are illustrated in Fig. 5 for the case of three uncertain parameters $\bar{\delta}_{1}, \bar{\delta}_{2}$, and $\bar{\delta}_{3}$, which are constrained by $\bar{\delta}_{1}=\bar{\delta}_{2}$. Thus $V_{1} V_{2}, V_{2} V_{8}$ are ce's, while $V_{1} V_{8}$ is not. Examples of gce's are edge $V_{1} V_{8}$ and face $V_{2} V_{3} V_{8} V_{7}$. Finally the image of the gce is crossed by the origin if $M_{1} M_{2}$ is crossed, and the image of the gce $V_{2} V_{3} V_{8} V_{7}$ is crossed by the origin if both arcs $M_{2} M_{3} M_{8}$ and $M_{2} M_{7} M_{8}$ are crossed.

We now state the counterpart of Theorem 1.

\section{Theorem 4}

We consider the multilinear mapping $\bar{p}(j \omega ; k \bar{\delta})$ defined for each $\omega$ in (3.4a) and the convex hull of the region $\bar{p}(j \omega ; k \bar{D})$ defined in $(3.4 \mathrm{~b})$. We increase $k$ from $k=0$ to the value $k=l$ for which the convex hull intercepts the origin for the first time. If this does not occur for $k<\infty$ then we obtain $k_{m}(j \omega)=\infty$. Otherwise for $k=l$ we can identify either a vertex $V_{i}$ of $\bar{D}$ which is mapped onto the origin, or two vertices $V_{i}$ and $V_{j}$ so that the origin lies on the line segment joining their images (critical vertices). In the former case and if $V_{i}$ satisfies (3.6) we obtain $k_{m}(j \omega)=l$. Otherwise we have $l \leq k_{m}(j \omega)$.

Next we increase $k$ to $k=u$ so that the origin crosses the image of a gce. If this does not occur for $k<\infty$, we set $u=\infty$. Then we have $k_{m}(j \omega) \leq u$.

Proof: The first part of the theorem relating to the lower bound on $k_{m}$ is shown as in the proof of Theorem 1. The second part relating to the upper bound on $k_{m}$ follows immediately, since if the origin crosses the image of a gce (see Defn. 4), it necessarily crosses the image of the corre- sponding constrained edge (see Fig. 5). This means that for $k=u$ a point $\bar{\delta}_{0}$ is found, which satisfies (3.6) and solves $\bar{p}\left(j \omega ; k \bar{\delta}_{0}\right)=0$. But then $k_{m}(j \omega) \leq u$ holds. If no such image is crossed $u=\infty$, and $u$ is trivially an upper bound.

\section{Step 4 (Modification of Theorem 2)}

Next we consider the computation of $k_{m}$ by subdividing $\bar{D}$ so that the bounds obtained in the previous theorem converge to $k_{m}$. Again for simplicity we assume that there is exactly one point $\bar{\delta}^{*}(j \omega)$ in $\bar{D}$ for each $\omega$, which satisfies $\bar{p}\left(j \omega ; k \bar{\delta}^{*}\right)=0$. In the following let $\delta_{i j}$ be a set of coordinates constrained by $\delta_{i s}=\delta_{i t}, s, t=1, . ., m(i)$. Let $h$ be one of the $2^{m(i)}$ $m(i)$-tuples $\left(h_{1}, . ., h_{m(i)}\right)$, where $h_{p}=1$ or $-1, \rho=1, . ., m(i)$. Define

$$
\begin{gathered}
\bar{D}_{h} \stackrel{\text { def }}{=}\left\{\vec{\delta} \in R^{m(1)} X \ldots X R^{m(n)} \mid-1 \leq \delta_{i j} \leq 1,\right. \\
i=1, \ldots, q-1, q+1, \ldots, n, j=1, \ldots, m(i), \\
\left.-1 \leq \delta_{q j} \leq 0 \text { if } h_{j}=-1,0 \leq \delta_{q j} \leq 1 \text { if } h_{j}=1\right\}
\end{gathered}
$$

Then we obviously have

$$
\bar{D}=\bigcup_{h} \bar{D}_{h}
$$

and we say that $(3.7 \mathrm{ab})$ define a partition of $\bar{D}$ along the edge $q$. Note that in (3.7a) only $\bar{D}_{1}$ and $\bar{D}_{2}$ corresponding to $h_{1}=(-1, \ldots,-1)$ and $h_{2}=(1, \ldots, 1)$ respectivily, contain the part of $\bar{D}$ that satisfies (3.6), and the rest of the $\bar{D}_{h}$ 's need not any further consideration. The situation is again illustrated in Fig. 5.

\section{Theorem 5}

We consider the mapping $\bar{p}\left(j \omega ; k_{m} \bar{\delta}\right)$ and the complex region $\bar{p}\left(j \omega ; k_{m} \bar{D}\right)$ defined by $(3.4 \mathrm{ab})$. Then there exists a partition of $\bar{D}$ as in (3.7ab), so that if we obtain bounds $l$ and $u$ for $\bar{D}$, $l_{11}$ and $u_{11}$ for $\bar{D}_{1}$, and $l_{12}$ and $u_{12}$ for $\bar{D}_{2}$ as in Theorem 4 and we set $u_{1}=\min \left\{u_{1 i}, u\right\}$, it holds

$$
l<l_{1 i} \leq k_{m} \leq u_{1} \leq u, i=1 \text { or } 2
$$

This process can be continued so that at the $r^{t h}$ step we obtain in general $s(r)$ subdomains of $\bar{D}$ with $s(r) \leq 2^{n}$ and corresponding bounds $l_{r i}, u_{r i}, i=1, \ldots, s(r)$, such that if we set $u_{r} \stackrel{\text { def }}{=} \min \left\{u_{r i}, u_{r-1}\right\}$, it holds

$$
l_{r i} \leq k_{m} \leq u_{r} \text {, for some } i=i^{*}, 1 \leq i \leq s(r)
$$

In this manner we obtain sequences $l_{r} \stackrel{\text { def }}{=} l_{r i^{*},}$ and $u_{r}, r=$ $1,2, \ldots$, such that

$$
\begin{gathered}
l_{r} \leq k_{m} \leq u_{r}, r=1,2, \ldots \\
l_{r}<l_{r+1}, \quad r=1,2, \ldots \\
l_{r} \longrightarrow k_{m}, \quad r \longrightarrow \infty \\
u_{r+1} \leq u_{r}, \quad r=1,2, \ldots
\end{gathered}
$$

and if $\left(k_{m}, \delta^{*}\right)$ is not an isolated real solution of (1.1)

$$
u_{r} \longrightarrow k_{m}, \quad r \longrightarrow \infty
$$

Proof: The proof parallels that of Theorem 2. We increase $k$ to $k=l$ so that $\operatorname{co}\{\bar{p}(j \omega ; k \bar{D})\}$ intercepts the origin and crit- 
ical vertices $V_{i}, V_{j}$ are defined and $V_{i} \neq V_{j}$ (see Theorem 4). We next consider a decomposition of $\bar{D}$ as in (3.7ab) so that $V_{i}$ and $V_{j}$ belong to different subdomains of $\bar{D}$. Then $\bar{\delta}^{*}$ is in $\bar{D}_{1}$ or $\bar{D}_{2}$, as in the discussion preceding Theorem 5 , and $l_{1} \stackrel{\text { def }}{=} l_{1 i^{*}}$ and $u_{1} \stackrel{\text { def }}{=} \min \left\{u_{1 i}, u\right\}$ satisfy (3.8), where $\bar{D}_{i^{*}}$, is the subdomain of $\bar{D}$, that contains $\bar{\delta}^{*}$. This procedure is repeated at the $r^{\text {th }}$ step for each subdomain obtained at the $(r-1)^{t h}$ step. If we let $l_{r} \stackrel{\text { def }}{=} l_{r i^{*}}, u_{r} \stackrel{\text { def }}{=} \min \left\{u_{r i}, u_{r-1}\right\}$ for $i^{*}$ such that $\bar{\delta}^{*} \in \bar{D}_{r i *}$, we obtain (3.9ab). If subdomains are divided so that critical vertices are separated (3.10) follows. At the $r^{t h}$ step subdomains $\bar{D}_{r i}$, that have $l_{r i}>u_{r-1}$ are dropped. We can prove (3.11) with an argument identical to the one used in proving Theorem 2. Finally (3.12) is obvious by construction and $(3.13)$ is proved by considering a sequence of subdomains $\bar{D}_{r}^{*}$, converging to $\bar{\delta}^{*}$ and reasoning as in the proof of Theorem 2.

\section{Remark}

We note that the modified algorithm retains the essential characteristics of the algorithm in $[5,6]$ and although it handles a more difficult problem, it requires a computational amount of the same order.

\section{Example}

For the system of (3.1), we take $\varsigma_{0}=0.15, \omega_{n 0}=3 \mathrm{rad} / \mathrm{sec}$, and $\left|\varsigma-\zeta_{0}\right| \leq 0.1,\left|\omega_{n}-\omega_{n 0}\right| \leq 0.5$. Our software implementing the algorithm of Section 3 at $\omega=3 \mathrm{rad} / \mathrm{sec}$ produced $k_{m}=1.5$ and revealed the most adverse parameter combination to be $\zeta^{*}=0.05$ and $\omega_{n}^{*}=3 \mathrm{rad} / \mathrm{sec}$. This result is in accordance with theoretical computations.

\section{Conclusions}

In this paper an algorithm was developed for the computation of the multiloop stability margin $k_{m}$ in the case of uncertain real parameters which can be related to each other. First, we showed that we can circumvent the rearrangement of the system to the diagonal canonical perturbation form of
Fig. 2. Secondly, we formulated the problem so that constraints among the parameters become the canonical constraints (3.6). Finally we modified the algorithm of Section 2 in order to account for the canonical constraints.

We remark that the algorithm presented effectively comprises a technique for finding the minimax solution of any polynomial equation in several real variables, and it is expected that it will be useful in many other situations.

\section{References}

[1] J. C. Doyle and G. Stein, "Multivariable Feedback Design: Concepts for a Classical/Modern Synthesis," IEEE Trans. Automat. Contr, vol. AC-22, pp 4-16, 1981.

[2] M. G. Safonov, A. J. Laub, and G. L. Hartmann, "Feedback Properties of Multivariable Systems: The Role and Use of the Return Difference Matrix,"IEEE Trans. Automat.Contr.,vol. AC-22, pp 47-65, 1981.

[3] M. G. Safonov, "Tight Bounds on the Response of Multivariable Systems with Component Uncertainty,"Proc. $16^{\text {th }}$ Allerton Conference, Monticello, Illinois, 1978.

[4] J. C. Doyle, "Analysis of Feedback Systems with Structured Uncertainty," I.E.E. Proc.,vol. 129, pt. D, no. 6, pp 242-250, 1982.

[5] R. R. E. de Gaston, Nonconservative Calculation of the Multiloop Stability Margin, Ph. D. Dissertation, University of Southern California, 1985.

[6] R. R. E. de Gaston and M. G. Safonov, "Exact Calculation of the Multiloop Stability Margin,"submitted to IEEE Trans. Automat. Contr., January 1986.

[7] L. Zadeh and C. A. Desoer, Linear System Theory, New York: McGraw-Hill, 1963.

[8] M. Vidyasagar, H. Schneider, B. Francis, "Algebraic and Topological Aspects of Feedback Stabilization," IEEE Trans. Automat.Contr., vol. AC-27, pp 125-129, 1892.

[9] J. R. Rice, The Approximation of Functions, Reading, MA: Addison-Wesley, 1969.

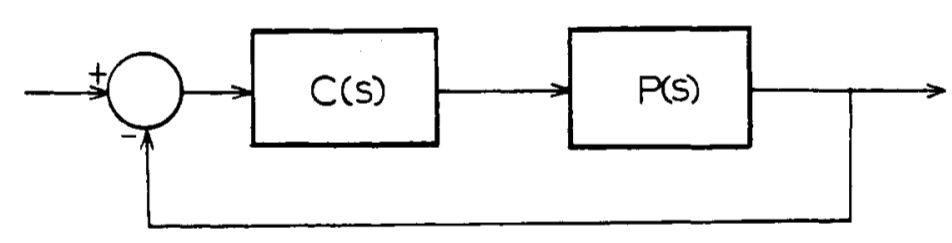

Figure 1

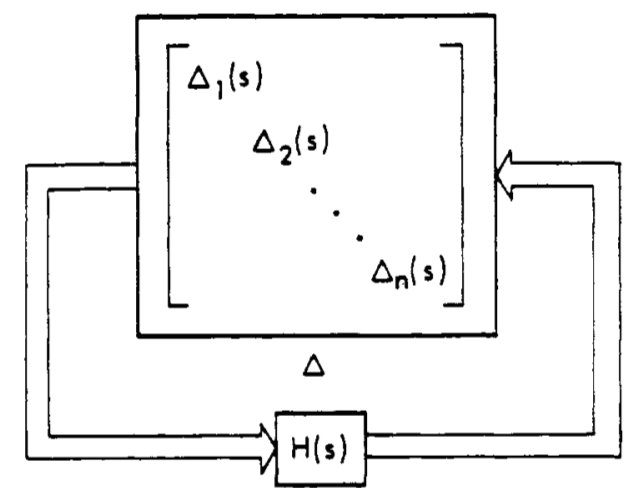




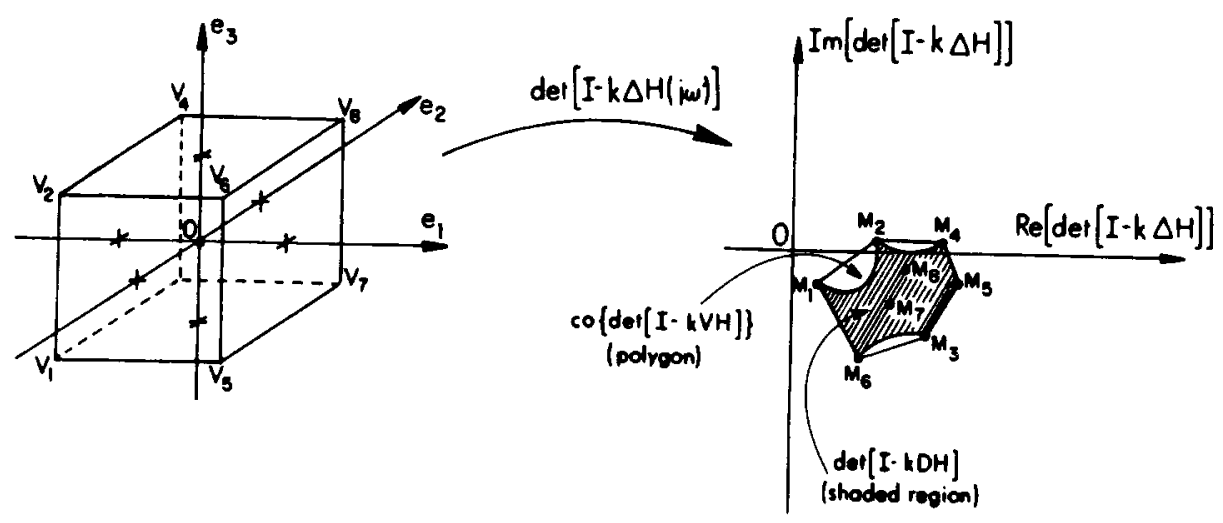

Figure 3a

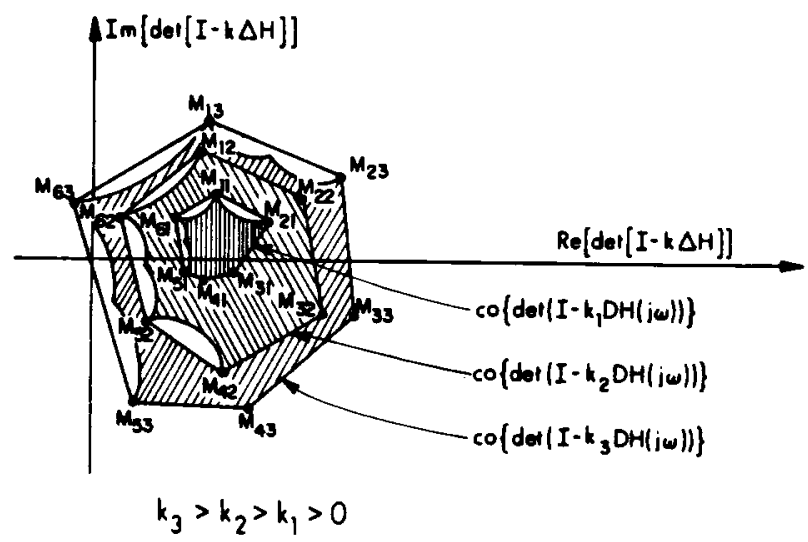

Figure 3b
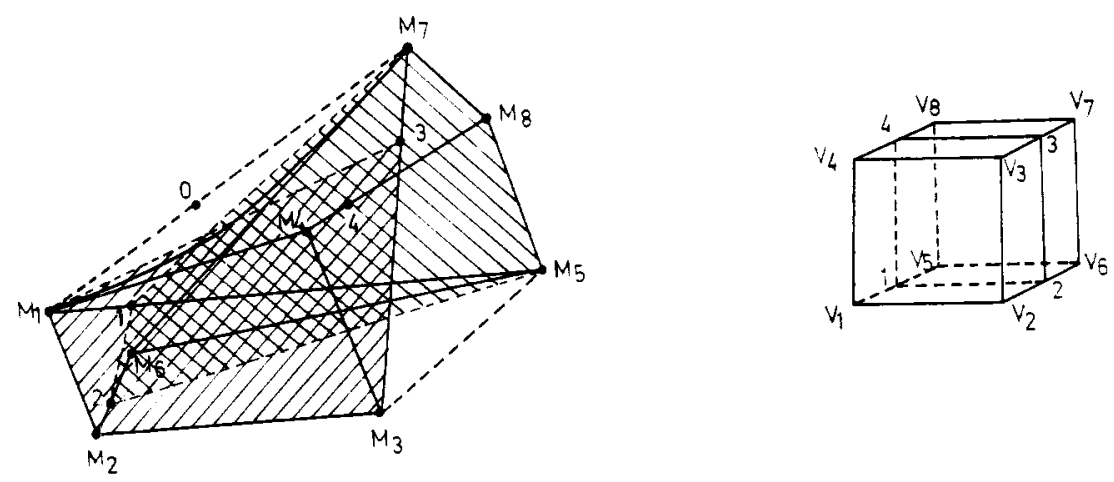

Figure 4
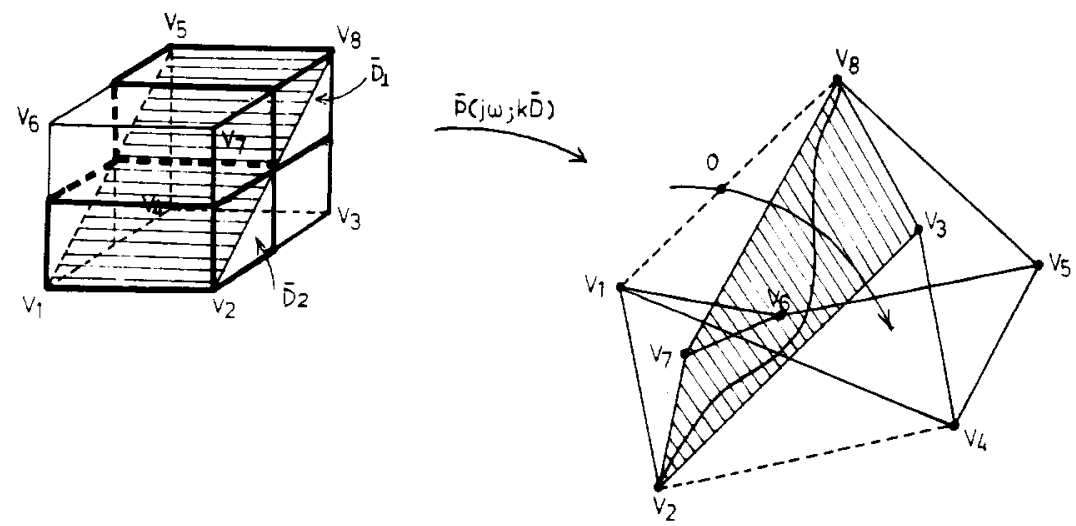

Figure 5 